\title{
Criminologie
}

\section{L'observation du comportement des jeunes en institution}

\section{Maurice Cusson}

Volume 8, numéro 1-2, 1975

Délinquance juvénile au Québec

URI : https://id.erudit.org/iderudit/017041ar

DOI : https://doi.org/10.7202/017041ar

Aller au sommaire du numéro

Éditeur(s)

Les Presses de l'Université de Montréal

ISSN

0316-0041 (imprimé)

1492-1367 (numérique)

Découvrir la revue

Citer cet article

Cusson, M. (1975). L'observation du comportement des jeunes en institution.

Criminologie, 8(1-2), 119-144. https://doi.org/10.7202/017041ar d'utilisation que vous pouvez consulter en ligne.

https://apropos.erudit.org/fr/usagers/politique-dutilisation/ 
L'OBSERVATION DU COMPORTEMENT DES JEUNES EN INSTITUTION

Maurice Cusson 
L'Observation du comportement des jeunes en institution ${ }^{2}$ l'(O.C.J.I.) est un instrument qui permet de décrire et d'analyser le comportement. Il est utilisé par les éducateurs qui ont eu l'occasion d'observer la conduite des jeunes.

L'O.C.J.I. rend possible la mesure de l'évolution d'un jeune au cours de son séjour dans des secteurs comme les relations avec ses camarades et avec ses éducateurs, la réaction à l'action éducative, les activités, etc.

Cet instrument se présente un peu comme un test qui peut être rempli rapidement par un éducateur mais, contrairement à la majorité des tests qui donnent une image statique des individus, l'O.C.J.I., utilisé périodiquement, permet de rendre compte de l'évolution des individus. A ce titre, il s'inscrit au cour même des préoccupations du véritable éducateur qui a appris à considérer le jeune mésadapté comme quelqu'un qui peut changer et qu'il doit aider dans sa croissance personnelle.

L'.O.C.J.I. a été conçu en fonction des objectifs de la resocialisation ou de la rééducation. Il permet de savoir jusqu'à quel point les buts visés par les éducateurs et les jeunes ont été atteints

1. La première version de l'O.C.J.I. a été construite à l'Ecole MontSaint-Antoine au cours d'une recherche à laquelle ont participé Jacques Beauchamp et Suzanne Laflamme-Cusson. Cette recherche était subventionnée par lo ministère des Affaires Sociales du Québec. La version - Boscoville de l'O.C.J.I. a été mise au point dans le cadre d'une recherche dans cette institution dirigée par Marc LeBlanc, directeur du Groupe de recherche sur l'inadaptation juvénile. Carole Mailloux et Suzanne Laflamme-Cusson ont participé a la mise au point de cette version de l'instrument. Cette dernière recherche est subventionnée par le ministère des Affaires sociales du Québec, la Fondation Donner, lo ministère de la Santé et du Bien-Etre social au Canada et le Solliciteur général du Canada. 
et comment se situent les jeunes par rapport aux interventions des éducateurs.

Si l'O.C.J.I. est rempli de façon régulière, il permettra à l'éducateur de réaliser ce qu'en pédagogie on appelle "évaluation continue », qui est la vérification à intervalles réguliers des acquisitions d'un jeune pendant une période d'apprentissage.

Une telle évaluation est essentielle à l'éducateur : elle lui permet de savoir si ses interventions ont atteint le but visé, et, ipso facto, d'améliorer constamment la qualité de son action. En effet, sachant quand il réussit et quand il échoue avec un jeune, l'éducateur peut corriger ses erreurs. Cette rétroaction (feedback) permettra de combler une lacune courante dans le domaine de la resocialisation : le fait que les éducateurs ne savent pas si leurs interventions ont apporté les résultats désirés. Or, cette évaluation est nécessaire à celui qui veut améliorer l'efficacité de son action.

L'O.C.J.I. a été développé à l'École Mont-Saint-Antoine et au Centre Boscoville, deux institutions pour adolescents délinquants situées dans la région de Montréal. Au Mont-Saint-Antoine, où il a vu le jour, l'O.C.J.I. est utilisé par les éducateurs dans leur évaluation périodique des garçons. Les éducateurs remplissent et corrigent eux-mêmes l'instrument puis ils réfléchissent sur les données ainsi obtenues, afin de comprendre l'évolution du sujet et d'élaborer la stratégie d'intervention la plus apte à aider le garçon à poursuivre son évolution ou à démarrer dans les secteurs où l'O.C.J.I. a démontré qu'il n'évoluait pas.

À Boscoville, une version adaptée au contexte de cette institution a été mise au point dans une perspective de recherche évaluative. Cette version de l'O.C.I.J. est un des deux instruments utilisés pour mesurer l'évolution des jeunes pendant le processus de rééducation ${ }^{2}$.

\section{A. LES PRINCIPES DE BASE DE L'O.C.J.I.}

\section{LES SOURCES DE L'O.C.J.I.}

L'O.C.J.I. repose sur une conception des objectifs de la resocialisation qui s'est constituée à partir d'une réflexion sur les buts poursuivis par les éducateurs et sur les caractéristiques des jeunes délinquants. Notre point de départ est venu de la constatation

2. L'autre instrument est un indicateur Etape (Tessier 1974) qui a été élaboré dans une perspective psycho-éducative. 
que les éducateurs du Mont-Saint-Antoine et de Boscoville avaient des notions très intéressantes des objectifs à poursuivre dans le cadre de leur travail. Ces conceptions s'exprimaient verbalement mais elles ont aussi été explicitées dans les écrits sur les étapes. (Gendreau 1960 - Mont-Saint-Antoine 1971 - Tessier 1970). Or, lorsque nous confrontons les conceptions d'éducateurs sur les objectifs de la resocialisation avec les études criminologiques sur la personnalité du délinquant et sur les facteurs associés à la récidive, nous constatons une convergence et une complémentarité des perspectives; les éducateurs insistant sur les buts immédiats à poursuivre, compte tenu de leur conception des valeurs, les études criminologiques identifiant les principales lacunes des délinquants, ce qui permettrait d'en déduire certains objectifs prioritaires.

Nous avons alors tenté d'opérer la fusion de ces deux perspectives et d'élaborer une conception originale de la resocialisation conçue comme un système de buts et de moyens ${ }^{3}$. Pour construire l'O.C.J.I., nous sommes partis de cette théorie, nous en avons dégagé une série de buts à court terme portant sur les capacités que le jeune doit acquérir pendant le processus de resocialisation, puis nous avons créé des énoncés décrivant des comportements qui constituaient des indices de la réalisation de ces buts.

\section{LE COMPORTEMENT}

Le comportement actuel du jeune est une des réalités fondamentales de la resocialisation. Ce principe permet de rendre compte d'un aspect essentiel de l'O.C.J.I. Nous utilisons ici le terme comportement dans un sens relativement large : acte significatif et observable posé par un jeune dans la vie quotidienne de l'institution. Les comportements mesurés par l'O.C.J.I. peuvent être regroupés en quatre catégories.

1) Des gestes, des manières de réagir à des situations ou à l'action des autres (ex. : perdre le contrôle de soi devant une contrariété, accepter les suggestions de l'éducateur, rendre service).

2) L'expression manifeste de sentiments (ex. : le repentir, la sympathie).

3) Les résultats ou la conséquence de l'action du sujet (ex. : le fait de réussir dans une activité).

3. Cette conception de la resocialisation est développée plus en détail dans un livre qui vient de paraître : Cusson M. (1974), la Resocialisation du jeune délinquant, Montréal, Presses de l'Université de Montréal. 
4) Les conduites et les sentiments que le sujet provoque chez les autres (c'est le cas des dimensions «popularité » et « leadership » : il s'agit ici d'une catégorie spéciale qui porte, non pas sur le comportement qu'il suscite chez les autres).

Tous ces comportements ont un certain nombre de points communs importants : 1) ils sont significatifs ; 2) ils sont directement observables; 3) ils portent sur le vécu actuel du jeune en institution.

1) Les comportements qui constituent l'O.C.J.I. sont significatifs.

Ce sont des actes qui sont l'expression d'attitudes, de sentiments, ou ce sont des gestes qui ont une finalité quelconque. Il ne s'agit pas de gestes physiques (ex. lever le bras), mais de conduites qui ont un sens facilement saisissable et qui permettent de comprendre l'orientation du sujet à l'egard d'autrui, de son milieu social ou de ses activités. Ceci suppose que l'observateur (en l'occurrence, l'éducateur) puisse comprendre les gestes du jeune. Ceci est parfaitement possible sans que la fidélité de l'instrument soit compromise, car il est connu qu'un adulte normal est capable d'interpréter le sens de la plupart des gestes posés par ceux avec qui il vit.

2) S'il est vrai que l'O.C.J.I. suppose une certaine dose d'interprétation, nous avons cependant voulu qu'il soit constitué des comportements les plus directement observables parce que nous voulions qu'il soit une mesure exacte et fidèle du comportement du jeune.

Nous nous sommes efforcés d'éliminer tout ce qui aurait laissé place à la subjectivité de l'éducateur, à la spéculation ou à l'arbitraire. Le travail d'interprétation que l'éducateur est appelé à faire est relativement simple.

3) Les comportements visés par l'O.C.J.I. portent sur le vécu actuel du jeune en institution. Ce sont des conduites qui montrent comment un pensionnaire réagit dans les conditions normales de la vie en institution. Il va sans dire qu'une telle vie est relativement artificielle. Mais, si un jeune s'y engage, elle peut devenir significative. Dans ce cas, il est important que l'éducateur connaisse bien la situation et le comportement actuel du jeune pour établir un plan d'intervention adéquat. Souvent, on accorde une importance excessive à ce qui ne peut être changé chez un jeune, comme les causes lointaines de ses problèmes. On oublie alors 
d'intervenir là où il est encore possible de réaliser des transformations véritables : sur son vécu immédiat.

Certains pourront se demander si l'O.C.J.I. n'accorde pas une importance exagérée au comportement et, ceci, aux dépens d'aspects plus fondamentaux de la personnalité du jeune. Une telle remarque suppose implicitement que, pour resocialiser un jeune mésadapté, il faut commencer par plonger dans les profondeurs de sa vie intérieure. Une telle démarche peut être nécessaire à l'occasion mais, dans la plupart des cas, elle n'est pas utile et elle détourne l'éducateur des problèmes sur lesquels il est possible d'agir. L'inadaptation et la délinquance sont fondamentalement des problèmes de comportements inappropriés qui conduisent le sujet à entrer en conflit avec son milieu. Le but des interventions de l'éducateur sera donc de transformer de façon durable le comportement du jeune. L'O.C.J.I. lui sera utile pour savoir s'il a réussi cette transformation.

Malgré l'importance que nous accordons aux comportements mesurés par l'O.C.J.I., nous ne prétendons pas qu'ils soient les seuls éléments dont l'éducateur doit tenir compte. Les conflits internes du jeune, son niveau de maturité interpersonnelle ou, chez quelques-uns, les problèmes psycho-pathologiques peuvent à juste titre retenir l'attention de l'éducateur.

L'O.C.J.I. n'est pas conçu pour appréhender de tels phénomènes. Il arrivera que les résultats de l'O.C.J.I. feront deviner à l'éducateur la présence de conflits ou d'une psycho-pathologie, mais il faudra avoir recours à d'autres méthodes ou à des spécialistes pour les évaluer de façon précise.

\section{LES OBJECTIFS DE LA RESOCIALISATION}

Selon nous, la resocialisation consiste avant tout en la démarche d'un jeune qui, incité par les éducateurs, poursuit des objectifs portant sur sa croissance personnelle. Quels sont ces objectifs? Il est possible d'en identifier trois.

a) Apprendre au jeune mésadapté à entrer en relation avec autrui.

b) Lui donner l'occasion de vivre une expérience d'insertion sociale véritable.

c) Le rendre autonome et capable d'affronter de lui-même les difficultés de la vie en société. 


\section{a) La qualité des relations avec autrui}

Très nombreux sont les criminologues qui s'entendent pour affirmer que l'on trouve, à la source de l'inadaptation sociale et de la délinquance, des problèmes de relations interpersonnelles ${ }^{4}$ : tendance à dévaloriser l'autre, incapacité de faire un effort pour conserver l'affection de quelqu'un, sentiment d'éloignement interpersonnel, indifférence affective, méfinance, égocentrisme, etc. Très souvent, ces problèmes de relation avec autrui sont le résultat de l'hostilité des gens qui vivaient avec le jeune et du rejet des parents.

La capacité d'établir avec d'autres personnes des relations de coopération et d'échange est un présupposé essentiel à toute adaptation sociale. C'est pourquoi, dans l'O.C.J.I., nous avons accordé une place de choix aux dimensions de relations interpersonnelles. L'éducateur pourra ainsi savoir si le jeune qu'il étudie évolue dans ses relations avec autrui, s'il fait les acquisitions désirables et s'il se corrige de ses comportements inadéquats dans ses rapports avec son entourage.

Les dimensions qui permettent de mesurer la qualité des relations avec autrui se subdivisent en "relations avec les pairs» qui comprennent la solidarité et l'agression et les « relations avec les éducateurs》 couvertes par l'ouverture et l'hostilité. À ceci s'ajoute une dimension qui concerne à la fois les relations avec les pairs et les relations avec les éducateurs, c'est la manipulation. Toutes ces dimensions permettent de vérifier des acquisitions d'un jeune dans la sphère des relations interpersonnelles : la capacité d'aider l'autre, d'éprouver de la sympathie, la capacité d'échanger. Les dimensions négatives permettent de vérifier si le jeune a réussi à résorber ou à éliminer des comportements désagréables ou menaçants : le fait de se battre, de provoquer ou d'utiliser autrui.

Il est possible de regrouper ces cinq dimensions (solidarité, agression, ouverture, hostilité, manipulation). De cette façon, nous construisons une dimension composée portant sur la qualité des relations. La définition opérationnelle de cette dimension serait :

L'ensemble des comportements qui manifestent que le sujet est positivement orienté à l'endroit d'autrui et qu'il entretient effectivement des relations interpersonnelles de qualité. La « relation» s'exprime par l'assistance à ceux qui en ont

4. Voir en particulier les auteurs suivants: Debuyst 1960, Pinatel 1963, Hirschi 1969, Fréchette 1970. 
besoin, par la sympathie, par le respect de l'autre, par la capacité d'échanger de façon franche et ouverte. Elle s'exprime aussi par l'absence de gestes qui tendent à nuire, à blesser ou à exploiter autrui.

\section{b) L'insertion sociale}

S'il est vrai que le jeune mésadapté éprouve de la difficulté à entrer en relation avec autrui, il n'est pas surprenant qu'il occupe une place marginale dans la société. Car c'est par l'intermédiaire des relations avec autrui que l'on participe à la vie collective. Le mésadapté, refusant de reconnaître l'autre, en arrive à refuser de participer à la vie collective et de se plier aux exigences du groupe. De leur côté, les membres du groupe se refusent à accepter cet individu qui ne répond pas à leur attente et ils en arrivent à vouloir l'exclure, accentuant ainsi sa marginalité.

Un des objectifs de la resocialisation sera donc d'amener le jeune à s'intégrer à la société et à respecter ses exigences. Or, un individu ne s'intègre pas à la société en général, il s'intègre à un ou à plusieurs groupes de dimension réduite qui sont des intermédiaires entre la société globale et l'individu. Dans le cas de jeunes en institution, le pavillon ou le quartier est ce groupe dans lequel le jeune pourra s'intégrer. Il est vraisemblable que, lorsque le jeune aura appris à participer à la vie sociale de son pavillon, il pourra, à son retour dans la société, s'intégrer et participer à la vie d'autres groupes.

Quatre dimensions permettent à l'éducateur de savoir jusqu'à quel point un jeune est intégré dans son groupe : l'intégration proprement dite, qui indique jusqu'à quel point le jeune apprécie le milieu où il se trouve, en particulier son pavillon, le leadership, la popularité et le statut qui permettent de savoir si le jeune est accepté par les garçons de son groupe.

\section{c) L'autonomie}

Il ne suffit pas que le jeune délinquant se réconcilie avec autrui et avec la société, encore faut-il qu'il puisse apporter à son milieu une contribution personnelle, qu'il ait la force de surmonter les difficultés de la vie en société et qu'il sache résister aux incitations à la déviance qui émanent de son entourage. En un mot, il faut qu'il soit autonome ou, plus précisément, que ses bonnes dispositions soient soutenues par une capacité d'agir de façon autonome. 
Or, sur ce chapitre, il est connu que les délinquants récidivistes présentent de graves lacunes. Á l'école et au travail, ils sont instables et inefficaces; ils ne peuvent faire face à la frustration et abandonnent à la première difficulté. Ils sont dépendants, suggestibles, flottant à la dérive, à la merci des incitations du premier venu. (Grygier 1966, Second 1970)

S'il est un concept qui nous apparaît indispensable dans la resocialisation c'est celui d'autonomie. Il est d'ailleurs fréquemment évoqué par les éducateurs. Mais son contenu reste imprécis, mal défini. Cela se comprend : l'autonomie est une réalité complexe qui implique plusieurs qualités. Soulignons-en quatre :

1) L'initiative dans la poursuite de projets personnels. Celui qui est autonome s'est fixé des objectifs, il a des projets. « Il sait ce qu'il veut $\gg$. Il n'a besoin ni de directives ni de pressions extérieures pour chercher activement à réaliser ce vers quoi il tend. Il prend lui-même l'initiative.

2) L'indépendance. Un homme autonome peut résister aux pressions de ceux qui veulent le détourner de ses projets. Il n'a pas un besoin excessif des autres pour savoir ce qu'il doit faire. Tous les hommes sont soumis à l'influence d'autrui mais celui qui est indépendant n'est pas totalement perméable aux pressions extérieures. Il ne cède pas aux pressions qui risquent de le mettre en conflit avec ce qu'il veut être.

3) La force. *Un homme qui détermine sa conduite d'après la perspective la plus courte, le repas d'aujourd'hui et justement ce repas-là est le plus faible des hommes ». (Makarenko 1967, p. 609). La force, c'est la maîtrise de soi, ou, pour parler comme les psychanalistes, c'est le principe de réalité qui est la capacité de renoncer à la satisfaction immédiate de ses désirs. C'est la force qui permet de tolérer les frustrations, de surmonter les difficultés, de ne pas se décourager devant l'échec.

4) La compétence. Face à un problème à résoudre, celui qui ignore ce qu'il faut faire et comment il faut le faire ne peut être autonome; il a besoin de l'aide et des conseils des autres. Donc, pas d'autonomie sans compétence, sans une connaissance et un savoir-faire précis dans un champ d'activité donné. Car la compétence est toujours liée à un secteur limité : on n'est pas compétent en général mais compétent dans tel métier, dans telle discipline. Et si l'autonomie suppose la compétence elle est aussi limitée à des secteurs déterminés. 
Sept dimensions de l'O.C.J.I. permettent d'appréhender l'autonomie : l'initiative qui réfère au fait de poursuivre des projets personnels, l'indépendance et la dépendance qui portent sur cette capacité de résister à l'influence d'autrui ; l'intolérance à la frustration qui mesure négativement la qualité de force dont nous venons de parler et les dimensions sport métier et activités académiques qui tentent d'appréhender la compétence dans ces secteurs d'activités.

Ces sept dimensions peuvent être regroupées pour constituer une dimension composée, l'autonomie qui pourrait être définie comme suit :

L'ensemble des comportements qui indiquent que le sujet est capable de penser et de décider de lui-même, sans avoir besoin des autres. Le sujet autonome a la force de poursuivre des projets personnels sans en être détourné ni par les événements ni par les personnes. Sa compétence lui permet de réaliser ces projets.

\section{LES RÉACTIONS DU JEUNE}

\section{A L'INFLUENCE DU MILIEU}

L'O.C.J.I. ne sert pas seulement à savoir jusqu'à quel point un jeune a réalisé les objectifs de la resocialisation, il apporte aussi des indications sur la manière dont il réagit à l'action des éducateurs qui veulent le faire évaluer. Deux dimensions composées, à $\mathrm{Y}$ ' acceptation * et la * participation 》, apportent des informations en ce sens. Elles portent sur les réactions des sujets aux interventions éducatives et elles peuvent être considérées comme des conditions préalables à la réalisation des objectifs.

\section{a) L'acceptation}

Dans la resocialisation, la nature spécifique du travail de l'éducateur sera d'influencer le jeune de telle sorte que ce dernier soit conduit à transformer sa conduite. Pour ce faire, deux modes d'interventions nous semblent particulièrement cruciaux : l'incitation et l'évaluation. L'incitation est l'action de l'éducateur qui entraîne le jeune à poursuivre les objectifs de la resocialisation et qui le dirige en ce sens. L'évaluation est l'appréciation, positive ou négative, que l'éducateur fait de la conduite du garçon. Elle comprend l'approbation et la récompense et, au pôle négatif, la désapprobation et la peine. Cependant, pour que les éducateurs exercent effectivement leur influence, encore faut-il que le jeune 
accepte cette influence, qu'il soit réceptif, perméable aux influences éducatives. Si le jeune accueille favorablement les interventions de l'éducateur, tous les espoirs sont permis. $\mathrm{Si}$, au contraire, le jeune se ferme et refuse de collaborer, l'éducateur restera impuissant. Voilà pourquoi l'" acceptation $\gg$ constitue un objectif à court terme essentiel : l'éducateur doit pouvoir faire accepter au jeune ses interventions. Cinq dimensions permettent de mesurer le degré d'acceptation : la conformité (la soumission à l'éducateur), la réaction à l'évaluation (le fait d'accepter les jugements et les sanctions infligés par les éducateurs), la réceptivité (la disponibilité devant l'influence de l'éducateur), la résistance (l'opposition à l'influence de l'éducateur) et l'acceptation de l'aide.

\section{b) La participation}

La participation est une forme plus avancée de réaction à l'influence éducative que «l'acceptation ». C'est une acceptation intériorisée. Il ne s'agit plus seulement pour le jeune de recevoir l'influence mais de s'engager activement dans le processus de resocialisation et d'apporter sa collaboration au milieu institutionnel. Ceci implique un choix du jeune à l'endroit de la resocialisation : il décide d'assumer les objectifs de la resocialisation. Deux dimensions de l'O.C.J.I. permettent de mesurer cette réalité : l'« engagement 》 (la décision de changer et de poursuivre les buts de la resocialisation) et la «contribution à la rééducation» (le fait d'aider ses camarades dans leur évolution).

\section{B. LA DYNAMIQUE DE L'O.C.J.I.}

Il nous reste maintenant à faire ressortir comment les dimensions composées de l'O.C.J.I. peuvent être reliées entre elles et comment elles peuvent jouer sur l'adaptation du jeune lors de son retour dans la société.

Le graphique qui suit présente schématiquement les principaux liens existant entre les dimensions ainsi que les liens entre ces dimensions et la non délinquance. Il s'agit ici de relations théoriques qui devront être vérifiées par l'étude des corrélations entre ces dimensions de même que par des études «follow up 》 établissant le lien entre ces dernières et la non-récidive.

Le lien entre la "relation avec les éducateurs 》 et l'« acceptation » (flèche 1) fait ressortir le fait que le jeune qui aura établi 


\section{GRAPHIQUE}

Liens entre les dimensions de lo.C.J.I. et leur influence sur la non-récidive

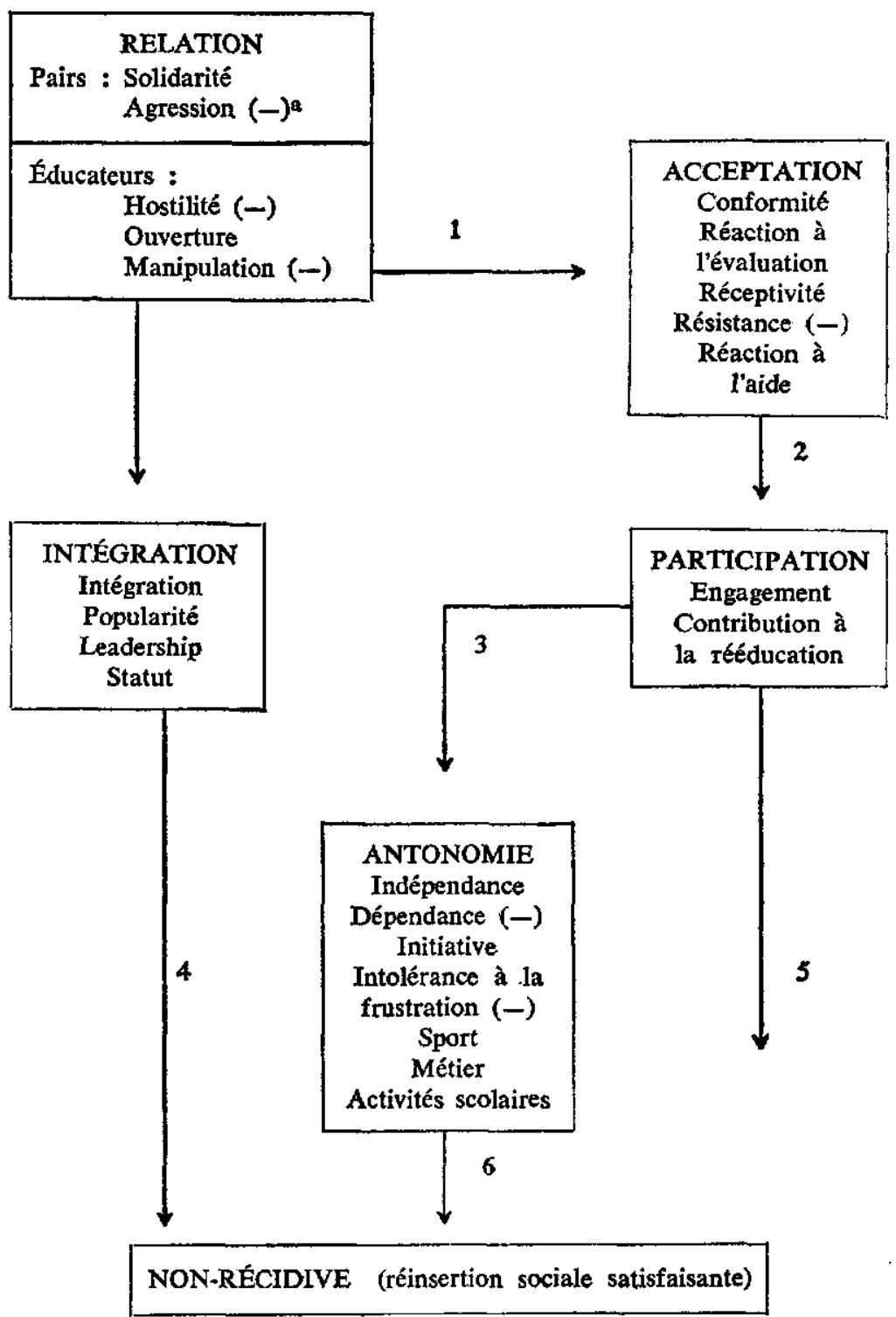

a Le signe (-) indique une dimension qui contribue négativement à l'ensemble de la dimension composée. 
des relations positives avec les éducateurs sera réceptif à leurs interventions et plus susceptible d'accepter leur influence.

L'\& acceptation 》 conduit à la «participation » (flèche 2) par un processus d'intériorisation de l'influence éducative : acceptant l'influence de l'éducateur, le jeune assimilera les principes que l'on veut lui inculquer, ce qui s'exprimera par un engagement dans le processus de rééducation et par la contribution au milieu.

Le sujet qui s'engage activement dans la rééducation risque de devenir plus « autonome » (flèche 3 ) : il a assimilé un système de buts qui le guide dans ses décisions et qui l'aide à résister aux influences susceptibles de le détourner de sa ligne de conduite. Cette relation repose sur une conception qui veut que l'éducation ait pour finalité d'aider l'enfant à développer sa nature propre, à s'affirmer et à le rendre capable d'être libre. C'est ce à quoi faisait allusion Gendreau (1974) lorsqu'il disait : "Dans le fond, la raison d'être des centres de rééducation, c'est de faire en sorte que les jeunes deviennent plus libres, libres face à eux-mêmes, face à cette société qui a été l'une des causes de leur inadaptation. ?

L'O.C.J.I. n'aura de sens que si les comportements qu'il mesure permettent de prédire une meilleure adaptation du sujet au terme de son séjour en institution. Ici se pose d'abord le problème de la stabilité, de la permanence des acquisitions qui ont été faites au cours du processus de resocialisation. Jusqu'à quel point les comportements appris par le jeune sont durables? Résisteront-ils aux difficultés qui attendent le sujet à sa sortie ? À ceci s'ajoute le problème que pose la différence entre un milieu institutionnel et un milieu social «normal ». Ce que le jeune apprend en institution est-il transposable dans un milieu différent? La réponse à cette question peut varier selon les institutions et selon les objectifs que l'on poursuit dans l'une ou l'autre institution. Pendant l'élaboration de l'O.C.J.I., un effort particulier a été fait pour rendre mesurables des objectifs qui portent justement sur des acquisitions transposables dans un milieu social normal. Il nous reste à vérifier jusqu'à quel point nous avons réussi. Dans l'immédiat nous pouvons indiquer comment les dimensions de l'O.C.J.I. nous paraissent théoriquement reliées à la non-récidive. 
L'« intégration » et la non-récidive (flèche 4) sont reliées entre elles selon l'hypothèse que celui qui a appris à s'intégrer dans un groupe pourra, par la suite, s'intégrer dans la société. Il aura le désir et la capacité de se faire accepter dans des groupes « normaux \ (famille, milieu de travail, etc...) Subissant alors l'influence de ces groupes, il sera supporté dans ses efforts pour respecter la loi.

Le lien entre la «participation $\gg$ et la non récidive (flèche 5) ne devrait pas poser de problème. Il est fort vraisemblable que celui qui décide d'abandonner son style de vie délinquant et de s'engager dans le processus de rééducation intériorisera les attentes de la société.

Il est permis de croire qu'il existe une filiation très étroite entre l'\& autonomie » et la non récidive (flèche 6). En effet, la combinaison de qualités que nous avons regroupées sous la rubrique autonomie devrait permettre au jeune de résister aux incitations à la délinquance venant des personnes ou des circonstances ; elle devrait l'aider à bien agir, même en l'absence de contrôle externe, et elle devrait le rendre capable d'apporter une contribution originale à son milieu.

Il faut ajouter ici une distinction qui nous apparaît importante. L'\& autonomie » est la capacité de décider de façon indépendante et d'avoir des projets personnels. Cependant, la nature de ces projets n'est pas précisée ici, non plus que l'utilisation qui sera faite de cette capacité de décider. On peut très bien imaginer un individu autonome qui poursuivra des projets illégaux et qui utilisera sa capacité de décider à des fins antisociales. En d'autres termes, on peut concevoir une autonomie « déviante \$ aussi bien qu'une autonomie «conformiste ». Et le délinquant autonome sera d'autant plus dangereux qu'il disposera de ressources personnelles plus grandes dans sa lutte contre la société. Par conséquent, on peut supposer que, seule, l'autonomie ne sera pas un facteur de resocialisation mais, ajoutée à la relation et à l'intégration, qui manifestent que le sujet s'est reconcilié avec son milieu, et à la participation, qui indique une intériorisation d'objectifs socialisés, l'autonomie devrait puissamment contribuer à la resocialisation du sujet. En d'autres termes, c'est à la condition que l'autonomie soit accompagnée d'une harmonisation du jeune avec son milieu qu'elle conduira à la resocialisation. 


\section{LA MISE AU POINT DE L'O.C.J.I.}

Pour mettre au point un instrument comme l'O.C.J.I., il est nécessaire d'entreprendre tout un travail d'expérimentation, de vérification et de contrôle. Nous décrirons brièvement ici les procédures utilisées dans la construction de l'O.C.J.I. ${ }^{5}$

Nous avons commencé par une série d'entrevues avec les éducateurs et les garçons des institutions concernées. Il s'agissait d'avoir l'information la plus précise sur l'évolution des jeunes, sur leurs comportements et sur la manière dont ils vivaient le système des étapes. Nous avons rencontré des garçons qui se situaient à différentes étapes du processus de resocialisation et nous les interrogions sur la vie dans l'institution : relation avec autrui, intégration dans le groupe, activités, réactions aux interventions éducatives, etc. Avec les éducateurs, les entrevues portaient sur la conduite des garçons selon leur niveau d'évolution, sur leurs conceptions des étapes, sur les objectifs de la resocialisation, sur les réactions des jeunes à leurs interventions, etc. Ces rencontres nous ont aidé à mieux comprendre la dynamique du comportement des jeunes en institution.

Ces informations, ajoutées aux notions théoriques déjà acquises, nous ont permis de créer une première version de l'O.C.J.I. (qui, à l'origine, comportait 350 énoncés).

Afin de savoir si les énoncés ainsi créés étaient bien adaptés à ce que nous voulions mesurer et s'ils décrivaient des réalités observables, nous avons demandé à des éducateurs de les évaluer en termes de clarté, de possibilités d'observation de fréquence et d'intérêt. Cette démarche nous a conduit à éliminer une série d'énoncés et d'en reformuler plusieurs. Par la suite, nous avons fait un pré-test qui nous a permis de rejeter les énoncés qui n'étaient pas assez clairs. Dans un dernier temps, nous avons fait une expérimentation de l'O.C.J.I. sur 163 sujets du Mont-SaintAntoine ${ }^{6}$. Les principales analyses des résultats de cette expérimentation ont été les suivantes : 1) Examen de la concordance des réponses de deux éducateurs qui remplissaient l'O.C.J.I. sur des sujets identiques afin de s'assurer de la fidélité interjuge des énoncés et de rejeter ceux qui n'étaient pas satisfaisants ; 2) Ana-

5. Une description détaillée de ces procédures a été faite dans les documents suivants : Beauchamp et al. (1973) - Cusson (1974 b-) S. Laflamme-Cusson (1974).

6. A Boscoville, cette étape n'est pas terminée encore. 
lyse des corrélations entre chacun des énoncés et la dimension à laquelle elle était sensée appartenir, ce qui nous a permis d'apprécier l'homogénéité de nos dimensions ; 3) Analyse factorielle afin de déterminer si les dimensions construites à partir des corrélations correspondaient aux facteurs qui ressortaient d'une analyse purement statistique. La confrontation des résultats de l'analyse factorielle et de l'analyse d'item nous a conduit à faire certains aménagements dans les dimensions et d'en regrouper quelques unes.

\section{CONCLUSION}

L'O.C.J.I. est encore dans une phase de développement et de mise au point. Il reste beaucoup à faire pour qu'il soit tout à fait satisfaisant. Il faudra entreprendre des études «follow-up » de sujets ayant été évalués à l'aide de l'O.C.J.I. afin de déterminer les dimensions qui sont associées à la non-récidive et pour identifier les profils d'évolution permettant de prédire une bonne réinsertion sociale.

Dans l'état actuel, l'O.C.J.I. est limité aux institutions où il a été mis au point ; il ne saurait être utilisé ailleurs sans modifications. Malgré ses limitations, l'O.C.J.I. peut déjà être utile. Sur le plan de la recherche, il permettra de faire des études longitudinales systématiques qui nous apprendront beaucoup sur l'évolution des jeunes en cours de resocialisation, qui pourront préciser la théorie des étapes et lui donner une base empirique.

Sur le plan de l'action, l'O.C.J.I. est dès maintenant utilisé par les éducateurs du Mont-Saint-Antoine pour analyser l'évolution de leurs élèves. Les informations ainsi recueillies peuvent servir de point de départ pour les éducateurs qui veulent connaître les effets de leurs interventions et qui veulent élaborer un plan d'action adapté au rythme personnel du jeune. 


\section{APPENDICE}

Les dimensions de l'O.C.J.I.

La version de 1'O.C.J.I. utilisée au Mont Saint-Antoine comporte 17 dimensions et 96 énoncés et la version de Boscoville couvre 14 dimensions et 95 énoncés. De nombreuses dimensions sont identiques dans les deux institutions. Certaines sont totalement différentes d'une institution à l'autre et dans quelques cas des réarrangements ont été opérés. Le lecteur remarquera qu'un certain nombre d'énoncés reviennent deux fois. C'est qu'ils appartiennent à des dimensions qui sont constituées du regroupement de deux dimensions ou encore ce sont des énoncés qui contribuent à deux dimensions.

\section{RELATIONS AVEC LES PAIRS}

\section{1 - Solidarité (Mont-Saint-Antoine et Boscoville)}

Définition : Le comportement du jeune qui aide ses camarades, respecte leurs droits et va au-devant de ceux-ci par sympathie

Enoncés :

- Il donne un coup de main aux nouveaux

- Il lui arrive d'offrir son aide spontanément à ses compagnons

- II est porté à aller parler aux nouveaux

- Il rend service à ses compagnons sans poser de conditions

- En cas d'offense faite à autrui, il manifeste du repentir

- Quand il est en chicane avec un camarade, il fait les premiers pas pour revenir en bons termes avec lui

- Il manifeste de la sympathie à ses compagnons qui sont mal pris

- Il fait montre d'indifférence aux droits et aux sentiments de ses camarades

- Il se moque des échecs et des erreurs des autres $(-)^{7}$

- Il veut être chum " avec tout le monde

- Il est intéressé à la vie de groupe

- 11 classe ses compagnons une fois pour toutes et rien ne le fait changer d'avis $(-)$

\section{2 - Agression (Mont-Saint-Antoine et Boscoville)}

Définition : Le comportement du joune qui se bat, agit avec violence, provoque ses compagnons et entretient une atmosphère propice aux manifestations de violence

\section{Enoncés :}

- Il provoque ses compagnons sans raison

- Il se chicane avec ses compagnons

- Il se bat avec ses camarades

- Il se sert de ses bras pour régler les conflits

- Il sème la chicane dans le groupe

- Il Iui arrive de faire preuve de violence inutile ou de cruauté

- Il se plaît à exprimer devant le groupe une opinion qu'il sait peu populaire

- Il respecte les opinions de ses compagnons qui sont différentes des siennes $(-)$

- Il se moque des échecs et des erreurs d'autrui

7. Les signes (-) indiquent les énoncés qui mesurent négativement la dimension et dont la cote doit être inversée à la correction. 


\section{RELATIONS AVEC LES ÉDUCATEURS}

3-Hostilité (Mont-Saint-Antoine et Boscoville)

Définition : Le comportement de l'élève qui refuse de coopérer avec les éducateurs et qui manifeste son opposition en les critiquant, en les discréditant, en les provoquant ou même en les insultant

Enoncés :

- Il s'oppose à ce que les éducateurs demandent

- Il se plaît à lancer des aftir. mations qui vont choquer les éducateurs

- Il se plaît à souligner les erreurs des éducateurs

- Il se plaît à e niaiser, les éducateurs

- Il laisse entendre que les décisions des éducateurs sont toujours mauvaises

- Il lui arrive d'insulter les éducateurs

4 - Ouverture (Boscoville)

Définition : Le comportement du garçon qui parlo de façon ouverte avec l'éducateur de ce qu'il est, et qui peut véritablement échanger avec lui

Enoncés :

- Quand il n'est pas d'accord avec les éducateurs, il en discute calmement avec eux.

- Il exprime ouvertement à l'éducateur ce qu'il pense et ce qu'il ressent

- Il lui arrive d'expliquer son comportement à l'éducateur

- Il parle de ses projets et de son avenir avec les éducateurs

\section{RELATIONS : PAIRS ET EDUCATEURS}

\section{5-Manipulation (Mont-Saint-Antoine et Boscoville)}

Définition : L'action du jeune qui utilise des moyens détournés ou fourbes pour obtenir ce qu'il désire et qui justifie ce qu'il fait de façon à ne jamais avoir tort

Enoncés :

- Il tente d'apitoyer les gens en jouant sur leurs bons sentiments

— Quand il est puni, il joue à l'innocent, à la victime

- Il flatte les éducateurs en vue d'avoir leurs faveurs

- Quand on lui reproche de manquer au règlement, il répond que tout le monde le fait

- Il est prêt à promettre n'importe quoi s'il $y$ voit son profit

- En cas d'échec, il se dit malchanceux

\section{INTÉGRATION}

6-Intégration (Mont-Saint-Antoine et Boscoville)

Définition : Le fait d'apprécier son quartier et de participer à son organisation et à son amélioration

\section{Enoncés :}

- Il donne son avis quand il s'agit de décorer ou d'arranger son pavillon

- Il accepte de remplir, pour le pavillon, une tâche qui

- Il porte des jugements négatifs sur son pavillon (-)

- Il défend son pavillon lorsqu'on l'attaque ou le critique n'est pas obligatoire 
7 - Popularité (Mont-Saint-Antoine)

Définition : Le fait d'être aimé, accepté, estimé par la majorité des membres du groupe et d'en éviter le rejet

Enoncés :

- Les autres aiment se tenir avec Iui

-Quand il parle, ses compagnons lui prêtent attention

- Lorsqu'on se divise en équipes, il est rapidement choisi

- Il peut tout dire de ce qu'il pense à ses compagnons sans crainte d'être rejeté

- Ses compagnons l'empêchent de s'exprimer $(-)$

- Quand un compagnon lui crée des ennuis, il y en a plusieurs pour le défendre

- Il est ridiculisé par ses compagnons (-)

8 - Leadership (Mont-Saint-Antoine)

Définition : Désigne l'influence qu'exerce un garçon sur ses camarades. Le leader est écouté et suivi quand il fait une suggestion ou quand il dit aux autres quoi faire

Enoncés :

- Quand il dit aux autres quoi faire, ils le font

- Quand il parle, les autres se taisent

- Quand il y a une décision à prendre pour le groupe, on lui demande son opinion plus souvent qu'à un autre

9- Statut (Mont-Saint-Antoine)

Définition : Le fait d'être accepté et d'être influent au sein du groupe. Le \& statut * est le produit de la fusion de deux dimensions : le "leadership " et la a popularité ». Cette dimension indique la place qu'occupe un garçon dans la hiérarchie du groupe, son degré d'influence et l'estime dont il jouit auprès de ses camarades

Enoncés :

- Quand il dit aux autres quoi faire, ils le font

- Les autres aiment se tenir avec lui

- Quand il parle, les autres se taisent

- Quand il y a une décision à prendre pour le groupe, on lui demande son opinion plus souvent qu'à un autre

- On le choisit souvent pour être chef d'une activité, d'une équipe

- Quand il parle, ses compa-

- On le choisit souvent pour être chef d'une activité, d'une équipe

gnons lui prêtent attention

- Lorsqu'on se divise en équipes, il est rapidement choisi

- Il peut dire tout ce qu'il pense à ses compagnons sans crainte d'être rejeté

- Ses compagnons l'empêchent de s'exprimer (-)

- Quand un compagnon lui crée des ennuis, il $y$ en a plusieurs pour le défendre

- Il est rídiculisé par ses compagnons (-)

- Il prend des initiatives

\section{ACCEPTATION}

10 - Conformité (Mont-Saint-Antoine et Boscoville)

Définition : Le fait de se soumettre à l'autorité de l'éducateur et de respecter les règlements de l'institution 
Enoncés :

- Il résiste tant qu'il peut quand les éducateurs l'obligent a faire quelque chose (-)

- Il se soumet facilement à Ia personne en autorité

- Il se conforme aisément au

- Il remplit sa fonction de ménage sans qu'on lui demande

- Il accepte et tient compte des suggestions des éducateurs

- Il accepte les punitions sans trop de difficulté règlement

\section{1 - Acceptation de l'aide (Boscoville)}

Définition : La conduite de l'élève qui accepte de se faire aider. Il demande l'aide et les conseils des éducateurs et profite du support qu'on lui apporte

Enoncés :

- Il refuse l'aide et le support que lui offrent les éducateurs $(-)$

- Quand il a besoin d'aide, il

la demande à l'éducateur

- Il accepte et tient compte des suggestions des éducateurs

\section{2 - Réaction à l'évaluation" (Boscoville)}

Définition : La conduite du garçon qui est sensible aux évaluations que les éducateurs font de son comportement. II accepte les sanctions qu'on lui inflige et modifie son comportement en conséquence

\section{Enoncés :}

-Il change son comportement en fonction des critiques de l'éducateur

- Quand il reçoit une sanction, il admet implicitement ou explicitement que l'éducateur a raison

- Il essaie de comprendre pourquoi on lui inflige une sanction

- Lorsqu'on le désapprouve, il améliore sa conduite

- Quand il reçoit une sanction, il se décourage et prétend qu'il lui est impossible de se conduire comme on voudrait $(-)$

- Il se choque ou proteste quand on sanctionne sa conduite $(-)$

- Il manifeste de l'indifférence aux sanctions et aux désapprobations (-)

-II accepte les punitions sans trop de difficultés

\section{3 - Réceptivité (Mont-Saint-Antoine)}

Définition : C'est le fait d'accepter l'influence des éducateurs, d'être disponible. Le jeune qui est réceptif s'ouvre aux éducateurs de ses projets et tient compte de leurs suggestions. Il assume les objectifs qui lui sont proposés

\section{Enoncés :}

- Il est capable de tenir compte de l'évaluation que les éducateurs font de son comportement

- Il fait des efforts pour atteindre les buts que les éducateurs lui suggèrent

8. Nous utilisons le mot évaluation dans le sens suivant : appréciation négative de la conduite du sujet pouvant aller jusqu'à la punition. Ceci comprend: les désapprobations, les critiques, les cotations faibles, les sanctions, les conséquences, les punitions. 
- Il accepte et tient compte des suggestions des éducateurs

- Avant d'entreprendre quoi que ce soit, il demande conseil aux éducateurs

- Il parle de ses projets et de son avenir avec les éducateurs

\section{4 - Résistance (Mont-Saint-Antoine)}

Définition : C'est le fait de s'opposer ouvertement à l'influence du milieu institutionnel. La résístance se manifeste par le refus hostile d'entrer en relation avec les éducateurs et par la contestation des exigences du milieu. Cette dimension est le résultat de la fusion des dimensions hostilité et conformité

\section{Enoncés :}

- Il se plaît à lancer des affirmations qui vont choquer les éducateurs

- Il se plaît à souligner les erreurs des éducateurs

- Il résiste tant qu'il peut quand les éducateurs l'obligent à faire quelque chose

- Il se soumet à la personne autorité (-)
- Il demande conseil aux éducateurs quand il a des problèmes personnels

- Quand il n'est pas d'accord avec les éducateurs, il en discute calmement avec eux
- Il s'oppose à ce que les éducateurs demandent

一 Il se plaît à * niaiser * les éducateurs

- Il laisse entendre que les décisions des éducateurs sont toujours mauvaises

- Il se conforme aisément au règlement $(-)$

\section{PARTICIPATION}

15 - Engagement (Boscovilie)

Définition : La conduite du garçon qui a décidé de changer. Ses efforts démontrent qu'il répond positivement aux éducateurs qui l'incitent à poursuivre des objectifs rééducatifs.

Enoncés :

- Il fait des efforts pour atteindre les buts que les éducateurs lui suggèrent

- Il a décidé de s'engager (de - s'embarquer ^) dans le programme-de l'institution

- Il prétend qu'il n'a pas l'intention de changer $(-)$

\section{6 - Contribution a la rééducation (Boscovilie)}

Définition : La conduite du garçon qui aide ses camarades à respecter les exigences du milieu, et à s'engager dans le processus de rééducation. Il aide les garçons en difficulté. Il facilite les relations entre éducateurs et élèves.

\section{Enoncés :}

- Il incite les gars à participer positivement à la vie du quartier

- Il lui arrive de dire aux gars du groupe que quelque chose ne se fait pas
- Il encourage les gars à produire dans les activités

- Il lui arrive de dire aux autres garçons que les conseils de l'éducateur sont valables 
- Quand un gars passe par une mauvaise période, il va jaser ou faire des choses avec lui pour lui donner un coup de main

- Il lui arrive de dire aux gars qu'il a changé pour le mieux depuis qu'il est à Boscoville
- Il lui arrive de prendre ouver. tement parti pour les éducateurs lorsqu'il y a désaccord entre ceux-ci et les gars

- Il lui arrive d'expliquer à l'éducateur le comportement d'un autre gars

\section{AUTONOMIE}

17 - Indépendance ${ }^{9}$ (Mont-Saint-Antoine et Boscoville)

Définition : Le comportement de l'élève qui peut penser, décider et agir par lui-même sans trop être influencé par ses camarades. Le garçon indépendant peut prendre position sans attendre que les autres le fassent

Enoncés :

- Il change facilement d'opinion sous l'influence des autres gars $(-)$

- Il a besoin de l'approbation des gars du groupe $(-)$

- Sa conduite dépend de celle des gars du groupe $(-)$.

- Il se laisse influencer facilement par les gars du groupe $(-)$

- En situation de conflit il prend position personnellement sans attendre la majorité

- Il dit son opinion à ses camarades même si celle-ci risque de leur déplaire

- Il décide ce qu'il a à faire sans demander l'opinion de ses camarades

18 - Dépendance (Mont-Saint-Antoine et Boscoville)

Définition : Le comportement de l'élève qui a constamment besoin de l'aide, des conseils et de l'approbation des éducateurs parce qu'il éprouve de la difficulté à décider et à agir par lui-même

\section{Enoncés :}

- Il tourne autour des éduca. teurs

- Il demande conseil aux éducateurs quand il a des problèmes personnels

- Il s'arrange pour toujours attirer l'attention des éducateurs

- Avant d'entreprendre quoi que ce soit, il demande conseil à l'éducateur

- Il demande de l'aide aux éducateurs à la moindre difficulté

- Il recherche manifestement l'approbation des éducateurs

\section{9 - Initiative (Boscoville)}

Définition : La conduite du garçon qui s'affirme en organisant ses temps libres de lui-même parce qu'il a des centres d'intérêt spécifiques et des projets personnels suffisamment réels et durables. Ce garçon a des idées personnelles

\section{Enoncés :}

- Il perd son temps pendant ses temps libres parce qu'il ne sait que faire $(-)$

- Il exprime des idées personnelles dans les discussions

9. Au Mont-Saint-Antoine, cette dimension s'appelle c autonomie s. 
- Il a un ou plusieurs centres d'intérêt qui l'occupent pendant ses loisirs

- Il organise ses temps libres à poursuivre des projets qu'il s'est fixés
- Il prend des initiatives

- Il sait s'occuper lui-même en fin de semaine

20 - Intolérance à la frustration (Mont-Saint-Antoine et Boscoville)

Définition : La capacité du jeune à garder le contrôle de lui-même sous l'impact de provocation ou de situations susceptibles de l'irriter

Enoncés :

- Il perd le contrôle de luimême à la moindre contra. riété

- Il accepte d'être critiqué ou contredit par ses compagnons sans se fầcher (-)

- Il se fâche immédiatement quand on l'agace

- Il réagit vivement quand on interrompt une activité qui l'intéresse

- La critique l'empêche de fonctionner

- Quand il fait quelque chose, il lui faut des résultats immédiats

21 - Sport (Mont-Saint-Antoine)

Définition : Le fait de participer aux activités sportives et d'y réussir

Enoncés :

— Les résutlats des compéti- - Il réussit bien dans les sports tions sportives sont impor- - Il se défile des parties (actitants pour lui

- Il s'exerce au sport dans ses temps libres vités sportives) en trouvant des excuses $(-)$

22 - Activités scolaires (Mont-Saint-Antoine)

Définition : Le comportement de l'élève qui s'applique en classe, fait preuve de persévérance, s'organise et qui, dans l'ensemble, réussit bien dans les matières académiques

\section{Enoncés :}

- Ses résultats sont importants pour lui

- Il travaille rapidement

- Il se laisse facilement distraire (-)

- Il refait les mêmes erreurs dans ses travaux $(-)$

- Il termine ses exercices scolaires

- Il sait organiser son travail

- Dans l'ensemble, il réussit bien

- Il est minutieux dans ses travaux

\section{$23-$ Métier (Mont-Saint-Antoine)}

Définition : Le comportement du jeune qui réussit bien dans les travaux qu'il exécute à l'atelier : il s'applique, sait organiser son travail, fait preuve de persévérance et travaille efficacement

Enoncés :

- Il s'applique dans les travaux qu'on lui demande de faire

- Il sait organiser un travail (avoir de la méthode)

- Il se laisse facilement distraire $(-)$

- Il accepte de recommencer le travail qu'il n'a pas réussi
- Il réussit bien dans son métier

-Il finit un travail qu'il a commencé

- Il apprend à partir de ses erreurs

- Il travaille vite

- Quand une difficulté se présente il abandonne (-) 


\section{BIBLIOGRAPHIE}

BEAUCHAMP, J., Suzanne LAFLAMME-CUSSON et M. CUSSON (1973) : Observation du comportement des jeunes en institution, rapport technique, Montréal, Ecole de Criminologie, Université de Montréal.

CUSSON, M. (1974) : A. la Resocialisation du jeune délinquant, Montréal, Les Presses de l'Université de Montréal.

CUSSON, M. (1974) : B. Observation du comportement des jeunes en institution, version Boscoville, Montréal, Groupe de recherche sur l'inadaptation juvénile.

DEBUYST, C. (1960) : Criminels et valeurs vécues : étude clinique d"un groupe de jeunes criminels, Louvain, Publications universitaires.

FRECHETTE, M. (1970) : « Le criminel et l'autre ^, Acta criminologica, 3, p. $11-102$.

GENDREAU, G. (1960) : Les étapes de la rééducation d'après l'expêrience de Boscoville, Montréal, Conférence présentée au 1er Colloque de recherche sur la délinquance et la criminalité.

GENDREAU, G. (1974) : * Doit-on fermer les institutions pour jeunes délinquants? Criminologie made in Canada, vol. II, $\mathbf{n}^{\circ} 1, \mathrm{p}$. 3-12.

GRYGIER, T. (1966) : Social adjustment, Personality and Behaviour in training schools in Ontario, Toronto, rapport présenté au Department of reform institution.

HIRSCHI, T. : Causes of delinquency, Berkeley, University of California Press.

LAFLAMME-CUSSON, Suzanne (1974) : O.C.J.I., Version Boscoville, Epuration de l'instrument à partir de l'étude de la concordance des jugements des éducateurs sur les énoncés et des distributions de fréquence des énoncés, Montréal Groupe de Recherche sur l'inadaptation juvnile.

MAKARENKO, A. (1967) : Euvres en trois volumes, Moscou, Editions du Progrès.

MONT SAINT-ANTOINE (1971) : le Mont Saint-Antoine centre de resocialisation, Montréal, Ecole Mont Saint-Antoine.

PINATEL, J. (1963) : Traité de droit pénal et de criminologie, Tome III, Paris, Dalloz. 
SECOND, P. (1970) : «Un inventaire de personnalité à fondement psychanalytique : *The Dynamic Personality * de T.G. Grygier, Annales de Vaucresson. N. 8, p. 85-112.

TESSIER, B. (1974) : Indicateurs d'étapes, Montréal, Ecole de PsychoEducation. 